\title{
Interpreting the truncated pentagonal number theorem using partition pairs
}

\author{
Louis W. Kolitsch \\ Department of Mathematics and Statistics \\ The University of Tennessee at Martin \\ Martin, Tennessee, U.S.A. \\ lkolitsc@utm.edu
}

\author{
Michael Burnette* \\ The University of Tennessee at Martin \\ Martin, Tennessee, U.S.A. \\ micburn@ut.utm.edu
}

Submitted: Dec 18, 2014; Accepted: Jun 5, 2015; Published: Jun 22, 2015

Mathematics Subject Classifications: 11P81

\begin{abstract}
In 2012 Andrews and Merca gave a new expansion for partial sums of Euler's pentagonal number series and expressed

$$
\sum_{j=0}^{k-1}(-1)^{j}(p(n-j(3 j+1) / 2)-p(n-j(3 j+5) / 2-1))=(-1)^{k-1} M_{k}(n)
$$

where $M_{k}(n)$ is the number of partitions of $n$ where $k$ is the least integer that does not occur as a part and there are more parts greater than $k$ than there are less than $k$. We will show that $M_{k}(n)=C_{k}(n)$ where $C_{k}(n)$ is the number of partition pairs $(S, U)$ where $S$ is a partition with parts greater than $k, U$ is a partition with $k-1$ distinct parts all of which are greater than the smallest part in $S$, and the sum of the parts in $S \cup U$ is $n$. We use partition pairs to determine what is counted by three similar expressions involving linear combinations of pentagonal numbers. Most of the results will be presented analytically and combinatorially.
\end{abstract}

Keywords: Partitions, Euler's pentagonal number theorem, Partition pairs.

\section{Introduction}

Euler's pentagonal number theorem gives an easy recurrence for the number of partitions of $n$, denoted by $p(n)$. Namely,

$$
p(n)=\sum_{j=1}^{\infty}(-1)^{j+1}(p(n-j(3 j-1) / 2)+p(n-j(3 j+1) / 2))
$$

\footnotetext{
*The results in Section 3 are based on Michael Burnette's undergraduate research project at UT Martin. He is currently a graduate student at Tennessee Tech.
} 
where $p(k)=0$ if $k<0$. An interesting question is to determine how far off from $p(n)$ we are if we truncate this recurrence sum before we reach $n-j(3 j-1) / 2<0$ or $n-j(3 j+1) / 2<0$. In [1] Andrews and Merca answered this question when we stop the recurrence sum after an odd number of terms. In [3] Kolitsch gave an answer when we stop the recurrence sum with $p(n-1)+p(n-2)$. In Section 2 we will use generating functions to prove the general results. In section 3 we will interpret the results combinatorially.

\section{A Generating Function Proof}

If we define $B_{k}(n)$ for $k \geqslant 0$ to be the number of partition pairs $(S, T)$ where $S$ is a partition with parts greater than $k, T$ is a partition with $k$ distinct parts all of which are greater than the smallest part in $S$, and the sum of the parts in $S \cup T$ is $n$, then the generating function for $B_{k}(n)$ is given by

\section{Theorem 1.}

$$
\sum_{n=0}^{\infty} B_{k}(n) q^{n}=\frac{1}{(q ; q)_{\infty}} \sum_{j=k+1}^{\infty}(-1)^{j+k+1}\left(q^{j(3 j-1) / 2}+q^{j(3 j+1) / 2}\right) .
$$

As an immediate consequence of Theorem 1 and Euler's pentagonal number theorem we get

\section{Corollary 2.}

$$
p(n)+\sum_{j=1}^{k}(-1)^{j}(p(n-j(3 j-1) / 2)+p(n-j(3 j+1) / 2))=(-1)^{k} B_{k}(n)
$$

since

$$
\begin{aligned}
\sum_{n=0}^{\infty} B_{k}(n) q^{n} & =\frac{1}{(q ; q)_{\infty}} \sum_{j=k+1}^{\infty}(-1)^{j+k+1}\left(q^{j(3 j-1) / 2}+q^{j(3 j+1) / 2}\right) \\
& =\frac{1}{(q ; q)_{\infty}}\left((-1)^{k+1}(q ; q)_{\infty}-(-1)^{k+1}-\sum_{j=1}^{k}(-1)^{j+k+1}\left(q^{j(3 j-1) / 2}+q^{j(3 j+1) / 2}\right)\right) \\
& =(-1)^{k+1}-(-1)^{k+1}\left(\sum_{n=0}^{\infty} p(n) q^{n}\right)\left(1+\sum_{j=1}^{k}(-1)^{j}\left(q^{j(3 j-1) / 2}+q^{j(3 j+1) / 2}\right)\right) .
\end{aligned}
$$

Comparing coefficients of $q^{n}$, we get the desired corollary.

To prove Theorem 1 we note that for $j>k$ the generating function for partitions that fulfill the criterion to be a partition $\mathrm{S}$ as described above with smallest part $j$ is $\frac{q^{j}}{\left(q^{j} ; q\right)_{\infty}}$ and the corresponding generating function for partitions that fulfill the criterion to be a partition $\mathrm{T}$ as described above is $\frac{q^{k(j+1)+\left(\begin{array}{c}k \\ 2\end{array}\right)}}{(q ; q)_{k}}$. 
Thus

$$
\begin{aligned}
\sum_{n=0}^{\infty} B_{k}(n) q^{n} & =\sum_{j=k+1}^{\infty}\left(\frac{q^{j}}{\left(q^{j} ; q\right)_{\infty}} \cdot \frac{q^{k(j+1)+\left(\begin{array}{c}
k \\
2
\end{array}\right)}}{(q ; q)_{k}}\right) \\
& =\frac{1}{(q ; q)_{\infty}} \sum_{j=k+1}^{\infty} \frac{q^{k(j+1)+\left(\begin{array}{c}
k \\
2
\end{array}\right)+j}(q ; q)_{j-1}}{(q ; q)_{k}} \\
& =\frac{1}{(q ; q)_{\infty}} \sum_{j=0}^{\infty} \frac{q^{k(j+k+2)+\left(\begin{array}{c}
k \\
2
\end{array}\right)+j+k+1}(q ; q)_{j+k}}{(q ; q)_{k}} \\
& =\frac{1}{(q ; q)_{\infty}} \sum_{j=0}^{\infty} q^{\left(3 k^{2}+5 k+2\right) / 2}\left(q^{k+1}\right)^{j}\left(q^{k+1} ; q\right)_{j} \\
& =\frac{1}{(q ; q)_{\infty}} \sum_{j=1}^{\infty}(-1)^{j+1}\left(q^{(j+k)(3(j+k)-1) / 2}+q^{(j+k)(3(j+k)+1) / 2}\right) \\
& =\frac{1}{(q ; q)_{\infty}} \sum_{j=k+1}^{\infty}(-1)^{j+k+1}\left(q^{j(3 j-1) / 2}+q^{j(3 j+1) / 2}\right) .
\end{aligned}
$$

To rewrite

$$
\sum_{j=0}^{\infty} q^{\left(3 k^{2}+5 k+2\right) / 2}\left(q^{k+1}\right)^{j}\left(q^{k+1} ; q\right)_{j}
$$

as

$$
\sum_{j=1}^{\infty}(-1)^{j+1}\left(q^{(j+k)(3(j+k)-1) / 2}+q^{(j+k)(3(j+k)+1) / 2}\right)
$$

we are using identity 10 on page 29 in [2] with $x=q^{k}$.

If we define $C_{k}(n)$ for $k>0$ to be the number of partition pairs $(S, U)$ where $S$ is a partition with parts greater than $k, U$ is a partition with $k-1$ distinct parts all of which are greater than the smallest part in $S$, and the sum of the parts in $S \cup U$ is $n$ then we have the following theorem.

Theorem 3.

$$
\sum_{n=0}^{\infty} C_{k+1}(n) q^{n}=\sum_{n=0}^{\infty} B_{k}(n) q^{n}-\frac{q^{k(3 k+5) / 2+1}}{(q ; q)_{\infty}} .
$$

From the proof of Theorem 1 we have

$$
\begin{aligned}
\sum_{n=0}^{\infty} C_{k+1}(n) q^{n} & =\sum_{j=k+2}^{\infty}\left(\frac{q^{j}}{\left(q^{j} ; q\right)_{\infty}} \cdot \frac{q^{k(j+1)+\left(\begin{array}{c}
k \\
2
\end{array}\right)}}{(q ; q)_{k}}\right) \\
& =\sum_{j=k+1}^{\infty}\left(\frac{q^{j}}{\left(q^{j} ; q\right)_{\infty}} \cdot \frac{q^{k(j+1)+\left(\begin{array}{c}
k \\
2
\end{array}\right)}}{(q ; q)_{k}}\right)-\frac{q^{k(3 k+5) / 2+1}}{(q ; q)_{\infty}}
\end{aligned}
$$

which gives the desired result. As an immediate consequence of Theorem 3 we get 


\section{Corollary 4.}

$$
\sum_{j=0}^{k}(-1)^{j}(p(n-j(3 j+1) / 2)-p(n-j(3 j+5) / 2-1))=(-1)^{k} C_{k+1}(n) .
$$

This corollary follows immediately from Corollary 2 by observing that Theorem 3 gives

$$
C_{k+1}(n)=B_{k}(n)-p(n-k(3 k+5) / 2-1) .
$$

From Theorem 1 in [1] we get

\section{Corollary 5.}

$$
C_{k}(n)=M_{k}(n)
$$

where $M_{k}(n)$ is the number of partitions of $n$ where $k$ is the least integer that does not occur as a part and there are more parts greater than $k$ than there are less than $k$.

Corollary 6. For $k \geqslant 1$,

$$
\begin{gathered}
\sum_{j=0}^{k-1}(-1)^{j}(p(n-j(3 j+1) / 2)- \\
p(n-j(3 j+5) / 2-1))+(-1)^{k+1} p(n-k(3 k+5) / 2-1) \\
=(-1)^{k-1}\left(C_{k}(n)+D_{k}(n)\right)
\end{gathered}
$$

where $D_{k}(n)$ is the number of partition pairs $(S, T)$ where $S$ is a partition with parts greater than $k$ containing at least one part equal to $k+1, T$ is a partition with $k$ distinct parts all of which are greater than $k+1$, and the sum of the parts in $S \cup T$ is $n$.

This corollary follows immediately by observing that

$$
\sum_{n=0}^{\infty}\left(C_{k}(n)+D_{k}(n)\right) q^{n}=\sum_{j=k+1}^{\infty}\left(\frac{q^{j}}{\left(q^{j} ; q\right)_{\infty}} \cdot \frac{q^{(k-1)(j+1)+\left(\begin{array}{c}
k-1 \\
2
\end{array}\right)}}{(q ; q)_{k}-1}\right)+\frac{q^{k+1}}{\left(q^{k+1} ; q\right)_{\infty}} \cdot \frac{q^{k(k+2)+\left(\begin{array}{c}
k \\
2
\end{array}\right)}}{(q ; q)_{k}} .
$$

Corollary 7. For $k \geqslant 2$,

$$
\begin{gathered}
p(n)+\sum_{j=1}^{k-1}(-1)^{j}(p(n-j(3 j-1) / 2)+p(n-j(3 j+1) / 2))+(-1)^{k+1} p(n-(k+1)(3 k+4) / 2) \\
=(-1)^{k+1}\left(C_{k}(n)+E_{k}(n)\right)
\end{gathered}
$$

where $E_{k}(n)$ is the number of partition pairs $(S, U)$ where $S$ is a partition with one part equal to $k$ and all other parts greater than $k, U$ is a partition with $k-1$ distinct parts all of which are greater than $k$, and the sum of the parts in $S \cup U$ is $n$.

This corollary follows immediately by observing that

$$
\sum_{n=0}^{\infty}\left(C_{k}(n)+E_{k}(n)\right) q^{n}=\sum_{j=k+1}^{\infty}\left(\frac{q^{j}}{\left(q^{j} ; q\right)_{\infty}} \cdot \frac{q^{(k-1)(j+1)+\left(\begin{array}{c}
k-1 \\
2
\end{array}\right)}}{(q ; q)_{k}-1}\right)+\frac{q^{k}}{\left(q^{k+1} ; q\right)_{\infty}} \cdot \frac{q^{(k-1)(k-1)+\left(\begin{array}{c}
k-1 \\
2
\end{array}\right)}}{(q ; q)_{k-1}} .
$$




\section{A Combinatorial Look at Our Results}

In this section we will combinatorially verify the result observed from Theorem 3 that was used to prove Corollary 4 and the companion result that relates $C_{k}(n)$ and $B_{k}(n)$. These two relationships are stated in the next theorem.

Theorem 8. For $k>0$,

$$
\begin{aligned}
& \text { (i) } B_{k}(n)-p(n-k(3 k+5) / 2-1)=C_{k+1}(n) \\
& \text { (ii) } p(n-k(3 k+1) / 2)=C_{k}(n)+B_{k}(n) .
\end{aligned}
$$

To prove part $(i)$ of Theorem 8 we need to show how the partitions of $n-k(3 k+5) / 2-1$ bijectively correspond to the partition pairs $(S, T)$ for $n$ where $S$ is a partition with all parts greater than $k$ and $k+1$ is included as a part and $T$ is a partition into $k$ distinct parts greater than $k$. Given a partition $P=\left\{a_{1}, a_{2}, \ldots, a_{r}\right\}$ with $a_{1} \leqslant a_{2} \leqslant \cdots \leqslant a_{r}$ and $\sum_{i=1}^{r} a_{i}=n-k(3 k+5) / 2-1$, we will construct a partition pair $\left(\{k+1\} \cup\left\{a_{i}: a_{i}>\right.\right.$ $\left.k\},\left\{t_{1}, t_{2}, \ldots, t_{k}\right\}\right)$ by defining $t_{i}=(k+1+i)+\sum_{j=1}^{i} \alpha(k+1-j)$ where $\alpha(m)$ is the number of parts equal to $m$ in $P$. This gives a partition pair of the desired type since $k+1+\sum_{j=1}^{k}(k+1+i)=k(3 k+5)+1$ and $\sum_{i=1}^{k} \sum_{j=1}^{i} \alpha(k+1-j)=\sum_{a_{i} \in P, a_{i} \leqslant k} a_{i}$. Thus $B_{k}(n)-p(n-k(3 k+5) / 2-1)$ counts the number of partition pairs for $n$ of the type counted by $C_{k+1}(n)$.

We illustrate the correspondence used to prove part $(i)$ below:

Let $k=2$ and $n=25$. The partition $P=\{1,2,2,3,5\}$ corresponds to the partition pair $(\{3,3,5\},\{6,8\})$ and the partition pair $(\{3,4,6\},\{5,7\})$ corresponds to the partition $P=\{1,2,4,6\}$.

To prove part ( $i i)$ of Theorem 8 we need to show that the partitions of $n-k(3 k+1) / 2$ bijectively correspond to the partition pairs counted by $B_{k}(n)+C_{k}(n)$. Given a partition $P=\left\{a_{1}, a_{2}, \ldots, a_{r}\right\}$ with $a_{1} \leqslant a_{2} \leqslant \cdots \leqslant a_{r}$ and $\sum_{i=1}^{r} a_{i}=n-k(3 k+1) / 2$, we will define $t_{i}=(k+i)+\sum_{j=1}^{i} \alpha(k+1-j)$ for $i=1,2, \ldots, k$. If $t_{1}$ is less than the smallest part in $P$ that is larger than $k$, our partition pair will be given by $\left(\left\{t_{1}\right\} \cup\left\{a_{i}: a_{i}>k\right\},\left\{t_{2}, t_{3}, \ldots, t_{k}\right\}\right)$ (note if $k=1$ then $T=\{\}$ ). These partition pairs are counted by $C_{k}(n)$. If $t_{1}$ is greater than or equal to the smallest part in $P$ that is larger than $k$, our partition pair will be given by $\left(\left\{a_{i}: a_{i}>k\right\},\left\{t_{1}, t_{2}, t_{3}, \ldots, t_{k}\right\}\right)$. These partition pairs are counted by $B_{k}(n)$.

We illustrate the correspondence used to prove part (ii) below:

Let $k=2$ and $n=25$. The partition $P=\{1,1,1,2,3,5,5\}$ corresponds to the partition pair $(\{3,5,5\},\{4,8\})$ and the partition pair $(\{3,4,5,6\},\{7\})$ corresponds to the partition $P=\{1,1,1,4,5,6\}$.

We now present a combinatorial proof of Corollary 5 by showing how the partitions of $n$ counted by $M_{k}(n)$ bijectively correspond to the partition pairs counted by $C_{k}(n)$. Given a partition $P=\left\{a_{1}, a_{2}, \ldots, a_{u}, b_{1}, b_{2}, \ldots, b_{v}\right\}$ with $a_{1} \leqslant a_{2} \leqslant \cdots \leqslant a_{u}<k<b_{1} \leqslant$ $\left.b_{2} \leqslant \cdots \leqslant b_{v}\right\}, u<v$ and $\sum_{i=1}^{u} a_{i}+\sum_{i=1}^{v} b_{i}=n$, we will define $x_{i}=b_{v-u+i}+a_{i}$ for $i=1,2, \ldots, u$. The corresponding partition pair will be $(S, T)$ where the $k-1$ elements of $T$ are defined by $t_{j}=$ smallest value among the $x_{i}$ 's where $a_{i}=j$ for $j=1,2, \ldots, k-1$ and $S=\left\{b_{i}: i \leqslant v-u\right\} \cup\left(\left\{x_{i}: i=1,2, \ldots, u\right\}-T\right)$. 
We illustrate the correspondence used to prove Corollary 5 below: Let $k=3$ and $n=31$. The partition $P=\{1,1,2,4,5,5,6,7\}$ will be transformed to $\{5$, (6), 7, (9) $\}$ where the $t_{j}$ 's have been circled. The corresponding partition pair will be $(\{4,5,7\},\{6,9\})$. If we look at the bijection in the other direction and start with the partition pair $(\{4,5,5,6\}$, $\{5,6\})$ we will first transform it to $\{4,(5), 5,5,(6), 6\}$. This will then become the partition $\{1,1,1,2,2,4,4,4,4,4,4\}$ counted by $M_{3}(31)$.

We now define $N_{k}(n)$ for $k>0$ to be the number of of partitions of $n$ where $1,2, \ldots, k$ all occur as a part and there are more parts greater than $k$ than there are less than or equal to $k$. The following theorem holds.

Theorem 9. For $k>0, N_{k}(n)=B_{k}(n)$.

We can use a correspondence similar to the one used to prove Corollary 5 to prove Theorem 9. Given a partition $P=\left\{a_{1}, a_{2}, \ldots, a_{u}, b_{1}, b_{2}, \ldots, b_{v}\right\}$ with

$$
a_{1} \leqslant a_{2} \leqslant \cdots \leqslant a_{u}=k<b_{1} \leqslant b_{2} \leqslant \cdots \leqslant b_{v},
$$

$u<v$ and $\sum_{i=1}^{u} a_{i}+\sum_{i=1}^{v} b_{i}=n$, we will define $x_{i}=b_{v-u+i}+a_{i}$ for $i=1,2, \ldots, u$. The corresponding partition pair will be $(S, T)$ where the $k$ elements of $T$ are defined by $t_{j}=$ smallest value among the $x_{i}$ 's where $a_{i}=j$ for $j=1,2, \ldots, k$ and $S=\left\{b_{i}: i \leqslant\right.$ $v-u\} \cup\left(\left\{x_{i}: i=1,2, \ldots, u\right\}-T\right)$.

We illustrate the correspondence used to prove Theorem 9 below: Let $k=3$ and $n=31$. The partition $P=\{1,1,2,3,4,5,5,5,5\}$ will be transformed to $\{4,(6), 6,(7),(8)\}$ where the $t_{j}$ 's have been circled. The corresponding partition pair will be $(\{4,6\},\{6,7,8\})$. If we look at the bijection in the other direction and start with the partition pair $(\{4,4,5\}$, $\{5,6,7\})$ we will first transform it to $\{4,4$, (5), 5, (6), (7) $\}$. This will then become the partition $\{1,1,2,3,4,4,4,4,4,4\}$ counted by $N_{3}(31)$. 
As an immediate consequence of Theorem 9 we have

Corollary 10.

$$
p(n)+\sum_{j=1}^{k}(-1)^{j}(p(n-j(3 j-1) / 2)+p(n-j(3 j+1) / 2))=(-1)^{k} N_{k}(n) .
$$

\section{Concluding Remarks}

A natural question that arises from this paper is whether or not partition pairs can be used to interpret other truncated series. In particular, can they be used to answer question 2 posed by Andrews and Merca in [1]?

\section{References}

[1] Andrews, G. E. and Merca, M., The truncated pentagonal number theorem. J. Combin. Theory Ser. A 119 (2012), no. 8, 1639-1643.

[2] Andrews, G. E., The Theory of Partitions. Encyclopedia of Mathematics and Its Applications, vol. 2. Addison-Wesley, Reading (1976).

[3] Kolitsch, L. W., A connection between ordinary partitions and tilings with dominoes and squares. Integers 7 (2007), A10, 2 pp. 